UNITED STATES DEPARTMENT OF THE INTERIOR

GEOLOGICAL SURVEY

\title{
Principal Facts for 125 Gravity Stations in and around the Goshute Mountains-Toano Range, Elko County, Nevada
}

by

R. W. Saltus ${ }^{1}$ and R. N. Harris ${ }^{2}$

Open-File Report 86-153

1 U.S. Geological Survey, Denver, Colorado

2 U.S. Geological Survey, Menlo Park, California

Any use of trade names is for descriptive purposes only and does not imply endorsement by the USGS. 


\section{Table of Contents}

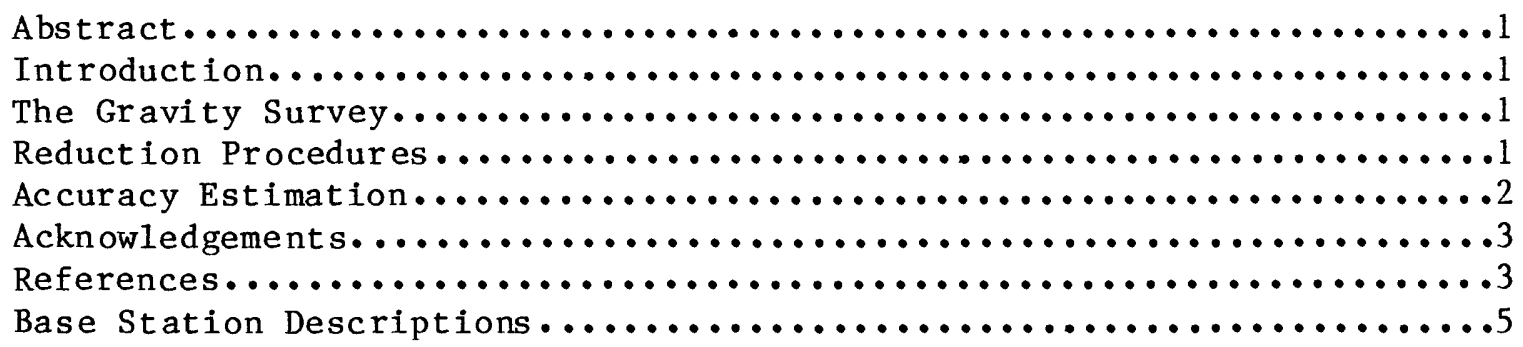

Tables

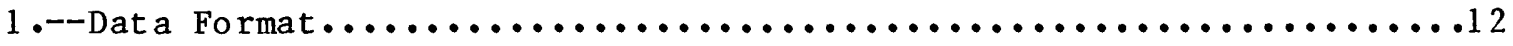

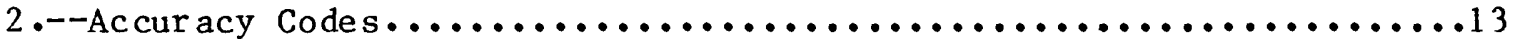

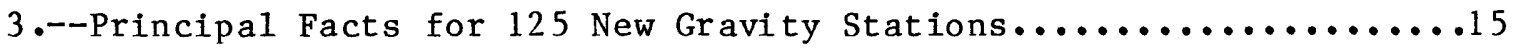

\section{Figures}

Figure 1.--Index Map of Gravity Station Locations................11 
Abstract

This report contains complete descriptions of 125 gravity stations located to the west of Wendover, Utah in the Goshute-Toano Range, E1ko County, Nevada. The stations were established in June, 1985, as part of a USGS/BLM wilderness appraisal study. Included in this report are procedures and equipment used, base station descriptions, accuracy estimates, and listings of reduced Bouguer anomaly and Airy isostatic residual anomaly values.

\section{Introduction}

The 125 stations described in this report were established by the authors in and around the Goshute Mountains-Toano Range, Nevada (see figure 1). These data were collected for a mineral survey report on two BLM wilderness study areas: Blue Bells and Goshute Peak, Nevada. These data will also become part of the "Complete Bouguer Gravity Map of Nevada - E1ko Sheet" to be published by the Nevada Bureau of Mines and Geology.

\section{The Gravity Survey}

The gravity stations described in this report were established between June 6 and June 20,1985. The areal extent of the survey is shown in figure 1. The stations are located in the Toano Range and the Goshute Mountains as well as the adjoining Steptoe, Antelope, and Goshute valleys.

The primary base for this survey was to have been ACIC base number 4646-1 (Jablonsky, 1974) in Wendover, Utah. However, the original base has been destroyed. A new location was established near the old base (see base station descriptions). This base was checked relative to the ACIC E1y base number 0390-2. In addition, five secondary bases were established and tied to the Wendover base.

The gravity stations were located mainly at bench marks or on labeled elevations on USGS 1:24,000 scale maps. Elevations for a few stations were estimated using two altimeters. The station accuracy code indicates the method of elevation control (table 2).

The stations were established on single day traverses involving driving and hiking. Each traverse began and ended at a primary or secondary base station.

LaCoste and Romberg gravity meters $G 17$ and $G 614$ were used for this survey.

\section{Reduction Procedures}

Observed gravity was obtained from the gravity meter readings using the calibration constants provided by the manufacturer as well as secondary factors established by the USGS. The secondary calibration factors used were 1.0025 for G17 and 1.00038 for G614. These values were then adjusted to the base station value assuming a linear drift between the first and last reading of each day. 
The base datum is the International Gravity Standardization Network of 1971 (Morel1i, 1974). Bouguer gravity anomalies were computed using the 1967 Geodetic Reference System formula for theoretical gravity at sea level (International As sociation of Geodesy, 1971) as implemented on a computer (Cordel1 and others, 1982). A Bouguer reduction density of $2.67 \mathrm{~g} / \mathrm{cm}^{3}$ was used. Terrain corrections were calculated for the region extending radially to $167 \mathrm{~km}$ from each station using a digital elevation model based on a 15 second geographic grid (P1ouff, 1977). Inner zone terrain corrections from the station to a distance of 63 meters (Hayford-Bowie zones A and B of Swick, 1942) were estimated in the field using tables based on slope, cone, and broken-slope elevation models. Terrain corrections for the radial area between $63 \mathrm{~m}$ and $590 \mathrm{~m}$ (Hayford-Bowie zones $\mathrm{C}$ and $\mathrm{D}$ ) were calculated using templates and 1:24,000 scale topographic maps for stations in areas of high local relief. A curvature correction for the deviation of the spheroidal earth from the Bougue $r$ approximation has also been added.

As the final step to the reduction process, a broad regional based on the gravitational effect of Airy isostatic roots (Simpson and others, 1983) was removed from the data. The resultant isostatic residual anomaly emphasizes the gravity effects of density distribution in the upper crust (Simpson and others, 1986). For the Airy isostatic model we assumed a sea level crustal thickness of $25 \mathrm{~km}$, a surface 1 load density of $2.67 \mathrm{~g} / \mathrm{cm}^{3}$, and a density contrast at depth of $0.4 \mathrm{~g} / \mathrm{cm}^{3}$

\section{Accuracy Estimation}

There are three potential sources of error for the reduced gravity values reported here:

1. Error in observed gravity

2. Elevation inaccuracy

3. Error in terrain correction

Imprecision in the observed gravity values may be due to operator error (mis-reading, meter not level), or mechanical problems with the meter itself, either continuous (drift), or catastrophic ( $t a r e$, thermostat failure, electrical malfunction). Systematic error in the observed gravity values is dependent on the accuracy of the primary base station, WEND, relative to IGSN 71. This station is located near the old ACIC base Wendover (非646-1), which had a reported accuracy of $\pm 0.1 \mathrm{mGal}$. The observed gravity for WEND is based on two ties to ACIC station 非390-2 in E1y, Nevada (IGSN 71 observed gravity value 979480.08$)$. The new base is assumed accurate to within $\pm 0.3 \mathrm{mGal}$.

Imprecision due to operator error or catastrophic mechanical failure is taken to be negligible for this survey. Imprecision due to mechanical drift can be estimated from the base station closures made each day. The maximum daily drift for this survey was $0.07 \mathrm{mGal}$. This corresponds to a linear drift of approximately $0.01 \mathrm{mGal} /$ hour. The imprecision is the difference between the true drift and the linear drift model. We estimate this to be at most equal to the value of the drift itself. If the worst deviation occurs at the midpoint in time between the two base readings, we estimate a maximum imprecision for observed gravity values to be $0.04 \mathrm{mGal}$. 
Elevation inaccuracies effect the reduction of observed gravity to simple Bouguer gravity. An elevation factor equal to approximately $.06 \mathrm{mGal} /$ foot $(.19 \mathrm{mGal} / \mathrm{m})$ is applied by the combination of the free air and Bouguer corrections. Benchmark elevations are assumed accurate to within $1 \mathrm{ft}$, topographic spot elevations are assumed to be accurate to within $5 \mathrm{ft}$, and altimetry estimates made for this survey are assumed accurate to within $20 \mathrm{ft}$, or $50 \%$ of the difference between control elevations, whichever is less. This elevation imprecision causes from less than \pm 0.06 to $\pm 1.2 \mathrm{mGal}$ of uncertainty, depending on the station elevation control.

The effect of latitude inaccuracy on the precision of results is assumed to be negligible.

The error in terrain correction is potentially the biggest source of error in the final results. These errors may be systematic to the survey due to a regional deviation of the topographic model from actual topography. This systematic error is difficult to estimate, but is probably less than $10 \%$ of the outer zone corrections. Inner zone terrain corrections for stations in areas of high relief can be very imprecise. We assume that the inner zone terrain corrections estimated for this survey are accurate to within $30 \%$.

The survey is assumed to have a systemmatic error in observed gravity of less than $\pm 1 \mathrm{mGal}$ relative to IGSN 71. The overall precision of the complete Bouguer anomalies is less than $\pm 2 \mathrm{mGal}$. However, the imprecision of individual stations with large inner zone terrain corrections may be as large as $\pm 5 \mathrm{mGal}$.

\section{Acknowledgements}

The authors wish to thank Keith Ketner, Warren Day, Maya Elrich, and Myra Vaag for logistical guidance and support in the field. H. Richard Blank assisted in initial phases of the survey.

\section{References}

Corde11, Lindrith, Keller, G. R., and Hildenbrand, T. G., 1982, Bouguer gravity map of the Rio Grande Rift, Colorado, New Mexico, and Texas: U.S. Geological Survey Geophysical Investigations Map GP-949.

International Association of Geodesy, 1971, Geodetic References System 1967: International Association of Geodesy Special Publication, no. 3, $116 \mathrm{p}$.

Jablonsky, H. M., 1974, World Relative Gravity Reference Network: Aeronautical Chart and Information Center Reference Publication No. 25.

Morelli, C., (ed•), 1974, The International Gravity Standardization Net 1971: International Association of Geodesy Special Publication, no. 4, $194 \mathrm{p}$.

P1ouff, Donald, 1977, Preliminary documentation for a FORTRAN program to compute gravity terrain corrections based on topography digitized on a geographic grid: U.S. Geological Survey Open-File Report 77-534, 45 p. 
Simpson, R. W., Jachens, R. C., and Blakely, R. J., 1983, AIRYROOT: A Fortran program for calculating the gravitational attraction of an Airy isostatic root out to $166.7 \mathrm{~km}$ : U.S. Geological Survey open-File Report 83-883, 66 p.

Simpson, R. W., Jachens, R. C., Blakely, R. J•, and Saltus, R. W., 1986, A new isostatic residual gravity map of the conterminous United States with a discussion on the significance of isostatic residual anomalies: Journal of Geophysical Research, in press.

Swick, C. H., 1942, Pendulum gravity measurements and isostatic reductions: U.S. Coast and Geodetic Survey Special Publication 232, 82 p. 
LOCAI GRAVITY BASE STATION

U.S. GEOLOGICAL SURVEY

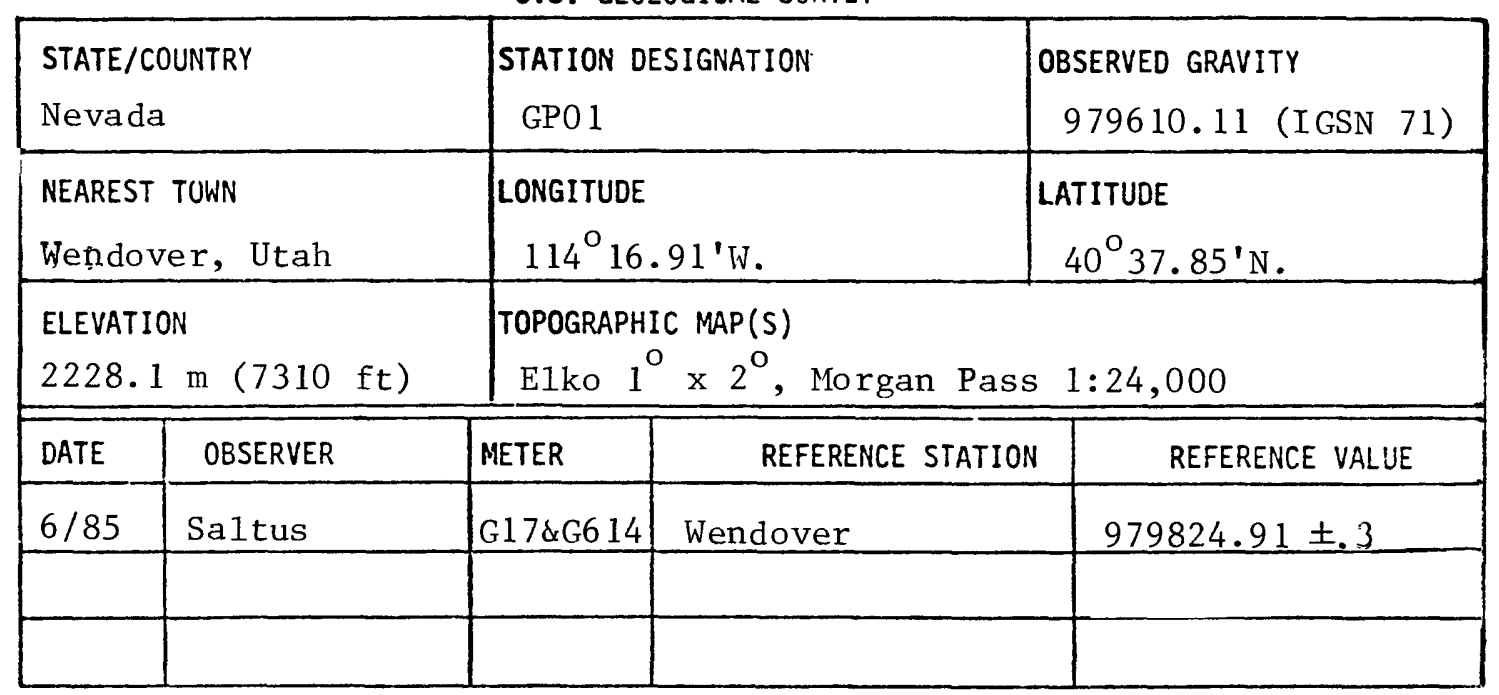

DESCRIPTION/SKETCH

This station is located at the top of Morgan Pass on the road separating the Toano Range from the Goshute Mountains. The station was read to the south of the road at its highest point, about 2 meters from the roadway. This station will be impossible to relocate exactly.

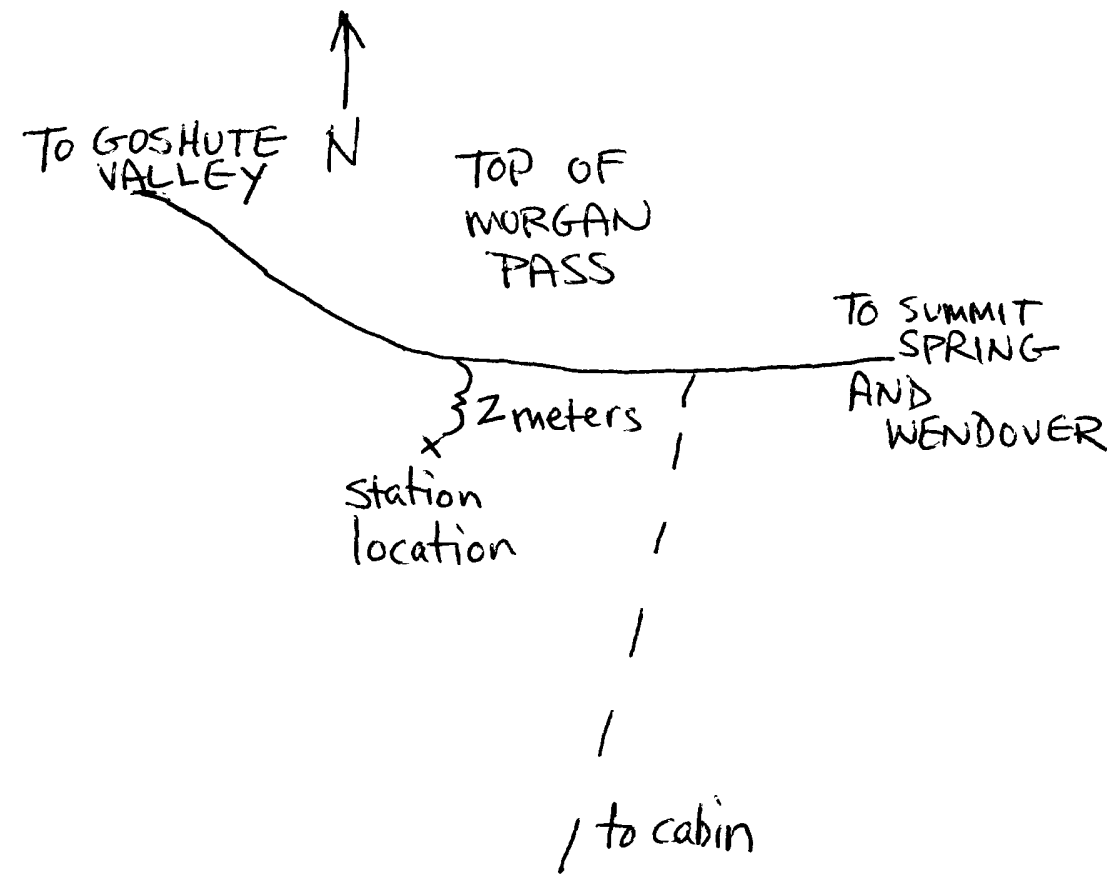




\begin{tabular}{|c|c|c|c|c|}
\hline \multicolumn{5}{|c|}{$\begin{array}{l}\text { GRAVITY BASE STATION } \\
\text { U.S. GEOLOGICAL SURVEY } \\
\end{array}$} \\
\hline \multicolumn{2}{|c|}{$\begin{array}{l}\text { STATE/COUNTRY } \\
\text { Nevada }\end{array}$} & \multicolumn{2}{|c|}{$\begin{array}{l}\text { STATION DESIGNATION } \\
\text { GV15 }\end{array}$} & $\begin{array}{l}\text { OBSERVED GRAVITY } \\
979686.47 \text { (IGSN 71) }\end{array}$ \\
\hline \multicolumn{2}{|c|}{$\begin{array}{l}\text { NEAREST TOWN } \\
\text { Wendover, Utah }\end{array}$} & \multicolumn{2}{|c|}{$\begin{array}{l}\text { LONGITUDE } \\
114^{\circ} 22.29^{\prime} \mathrm{W}\end{array}$} & $\begin{array}{l}\text { LATITUDE } \\
40^{\circ} 31.00^{\prime} \mathrm{N} .\end{array}$ \\
\hline \multicolumn{2}{|c|}{$\begin{array}{l}\text { ELEVATION } \\
1708.7 \mathrm{~m}(5606 \mathrm{ft})\end{array}$} & \multicolumn{3}{|c|}{$\begin{array}{l}\text { TOPOGRAPHIC MAP(S) } \\
\text { Elko } 1^{\circ} \times 2^{\circ} \text {, Lion Spring } 1: 24,000\end{array}$} \\
\hline DATE & OBSERVER & METER & REFERENCE STATION & REFERENCE VALUE \\
\hline $6 / 85$ & Saltus & G17\&G614 & Wendover & $979824.91 \pm .3$ \\
\hline & & & & \\
\hline
\end{tabular}

Located at benchmark labeled 13 FMK near Shafter well number 4. The meter was read on the ground directly to the north of the benchmark, with the baseplate touching the benchmark.

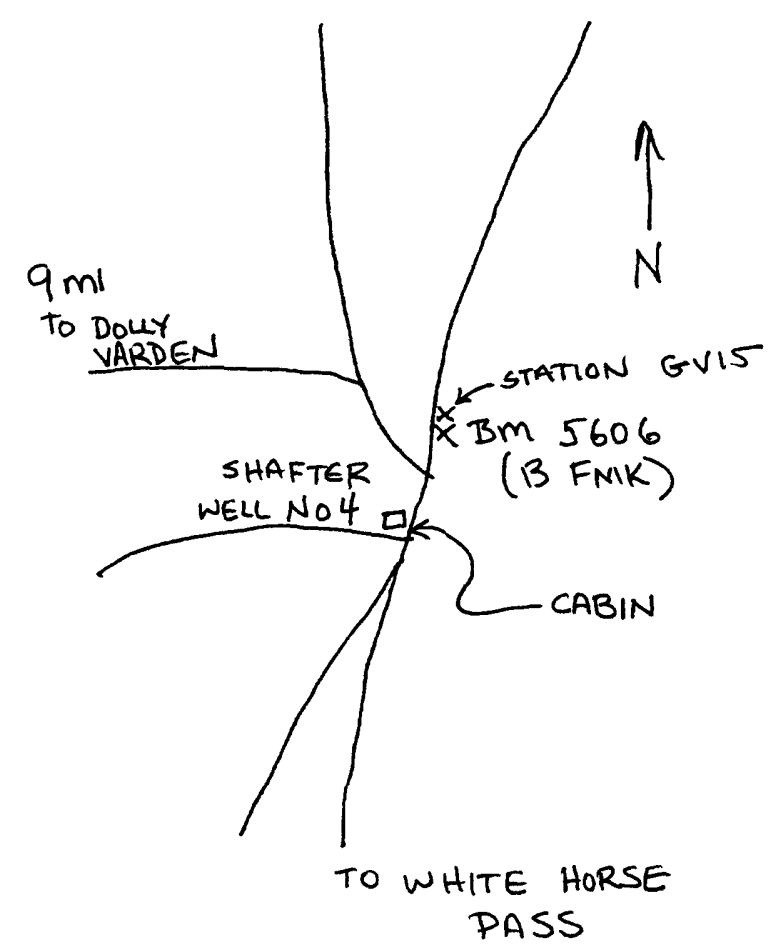


GRAVITY BASE STATION

U.S. GEOLOGICAL SURVEY

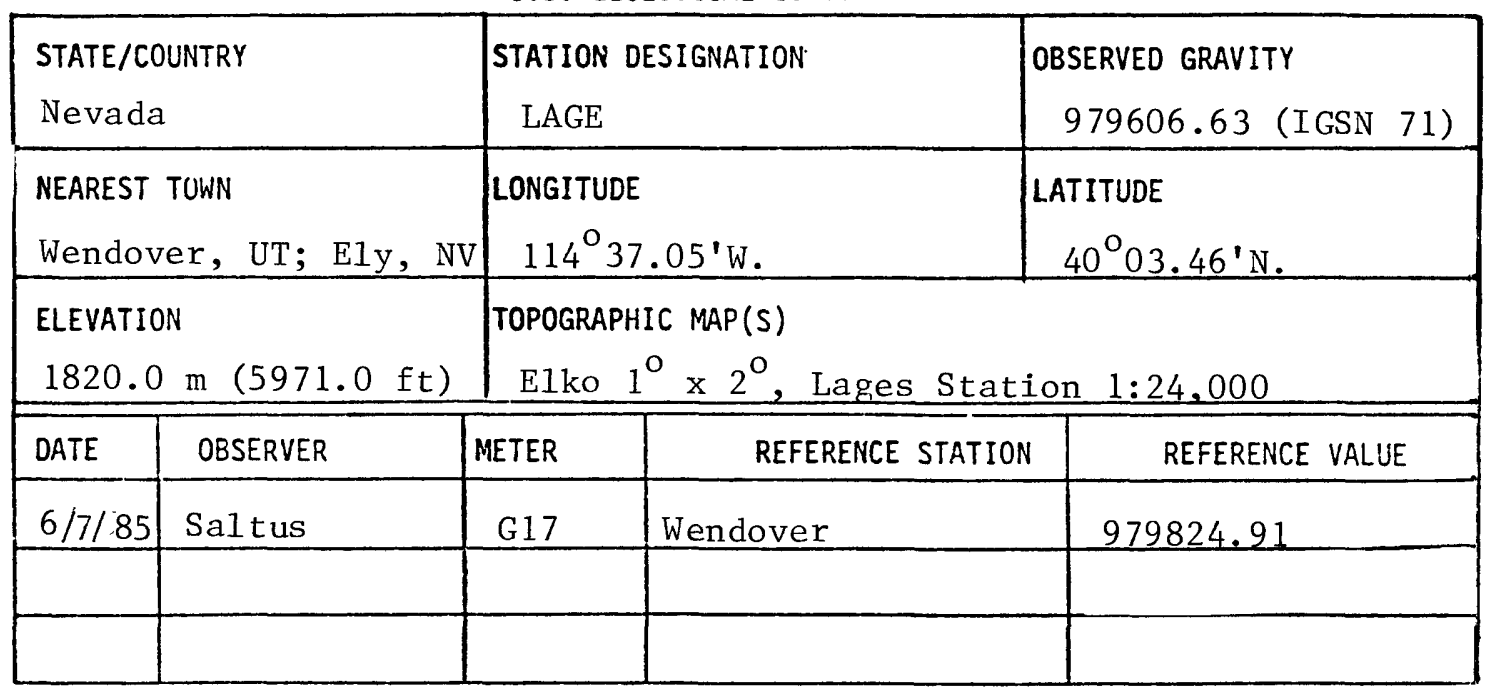

DESCRIPTION/SKETCH

The station is located on top of USC and GS benchmark R183 (1958) at southeast side of the Y-intersection of state highways ALT 50 and 93 halfway between Wendover, Utah and Ely, Nevada. The base plate is placed directly on the benchmark, about 5 inches above the ground.

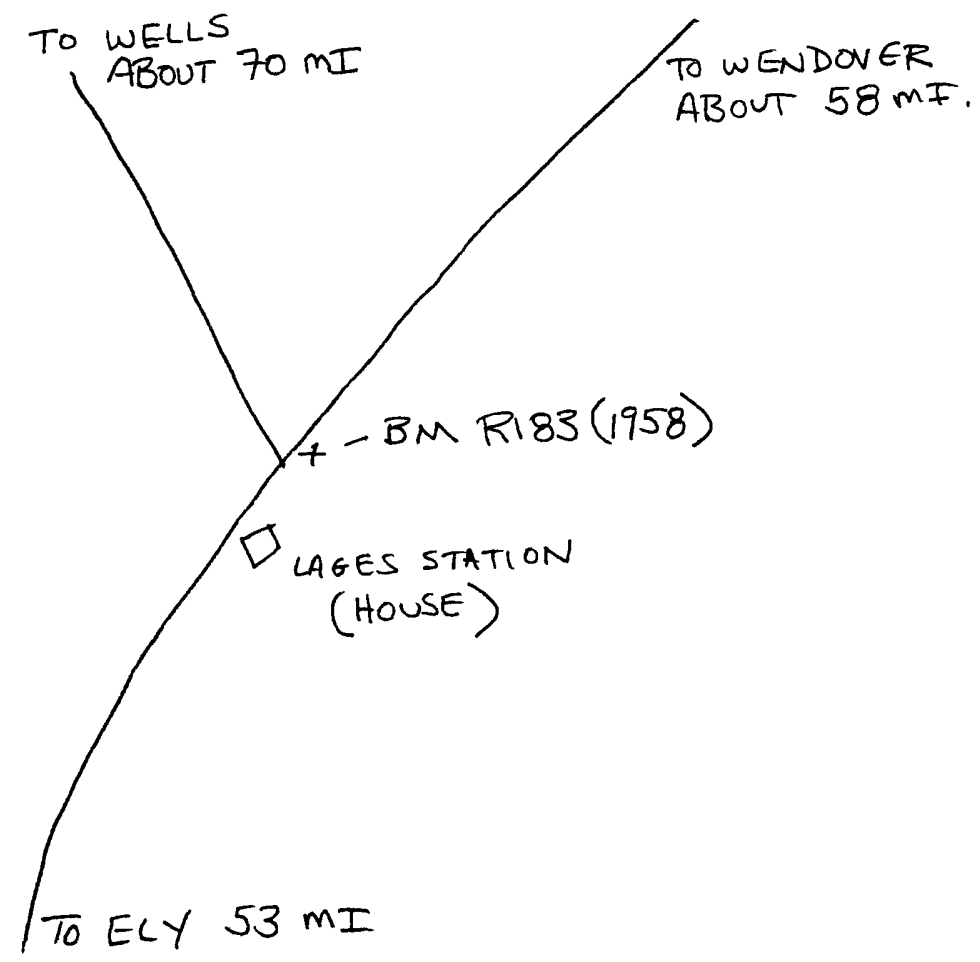


GRAVITY BASE STATION

U.S. GEOLOGICAL SURVEY

\begin{tabular}{|c|c|c|c|c|}
\hline \multicolumn{2}{|c|}{$\begin{array}{l}\text { STATE/COUNTRY } \\
\text { Nevada }\end{array}$} & \multicolumn{2}{|c|}{$\begin{array}{l}\text { STATION DESIGNATION } \\
\text { MORG }\end{array}$} & $\begin{array}{l}\text { OBSERVED GRAVITY } \\
979701.16 \quad \text { (IGSN 71) }\end{array}$ \\
\hline \multicolumn{2}{|c|}{$\begin{array}{l}\text { NEAREST TOWN } \\
\text { Wendover, Utah }\end{array}$} & \multicolumn{2}{|c|}{$\begin{array}{l}\text { LONGITUDE } \\
\qquad 114^{\circ} 20.65^{\prime} \mathrm{W} \\
\end{array}$} & $\begin{array}{l}\text { LATITUDE } \\
40^{\circ} 38.25^{\prime} \mathrm{N} \\
\end{array}$ \\
\hline \multicolumn{2}{|c|}{$\begin{array}{l}\text { ELEVATION } \\
1760.2 \mathrm{~m}(5775.0 \mathrm{ft})\end{array}$} & \multicolumn{3}{|c|}{$\begin{array}{l}\text { TOPOGRAPHIC MAP(S) } \\
\text { Elko } 1^{\circ} \times 2^{\circ} \text {, Morgan Pass 1:24,000 }\end{array}$} \\
\hline DATE & OBSERVER & METER & REFERENCE STATION & REFERENCE VALUE \\
\hline $6 / 7 / 85$ & Saltus & $\mathrm{G} 17$ & Wendover & 979824.91 \\
\hline $6 / 7 / 85$ & Saltus & G6 14 & Wendover & 979824.91 \\
\hline
\end{tabular}

DESCRIPTION/SKETCH

The station is located to the northeast of the northernmost intersection of the Morgan Pass road with the highest road skirting the eastern edge of Goshute Valley. In June, 1985, the location was $1 \mathrm{ft}$ south of a BLM "Wilderness Study Area" sign. The station is approximately $30 \mathrm{ft}$ E of and $2 \mathrm{ft}$ above the center of the road intersection marked 5773

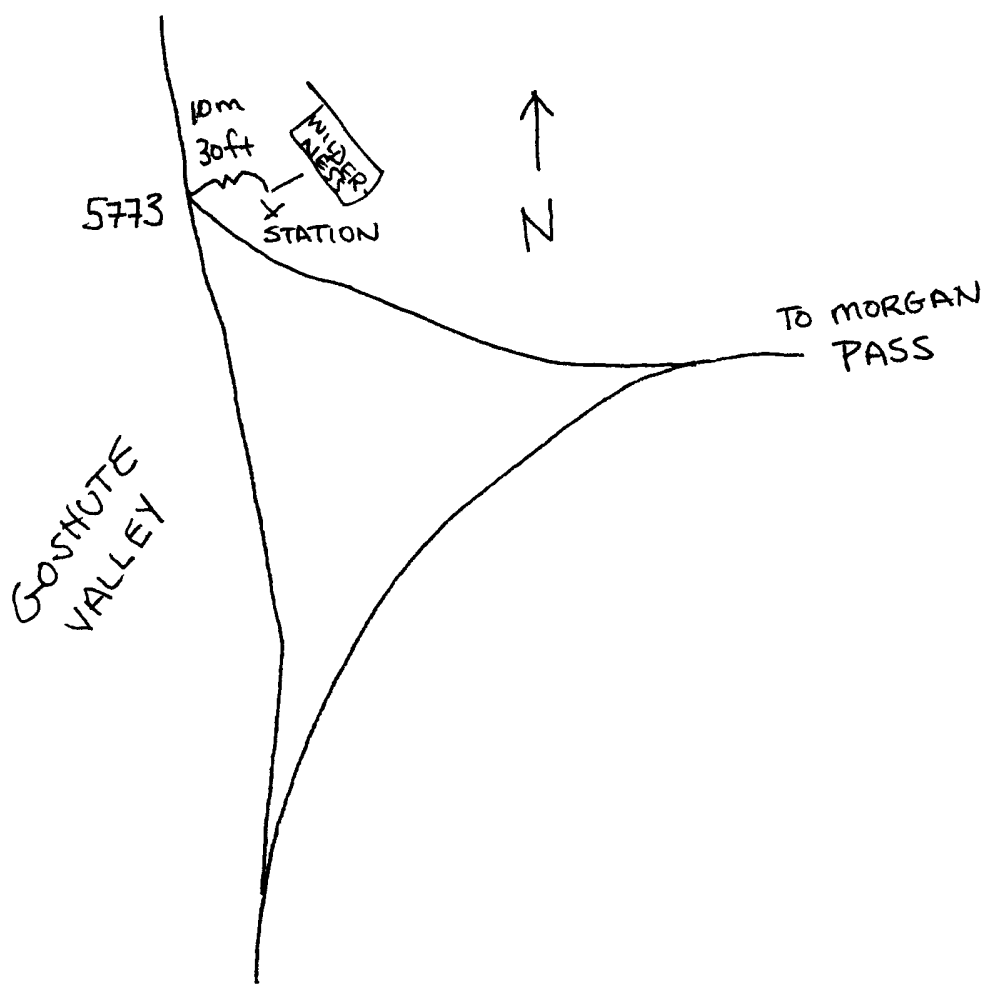


GRAVITY BASE STATION

U.S. GEOLOGICAL SURVEY

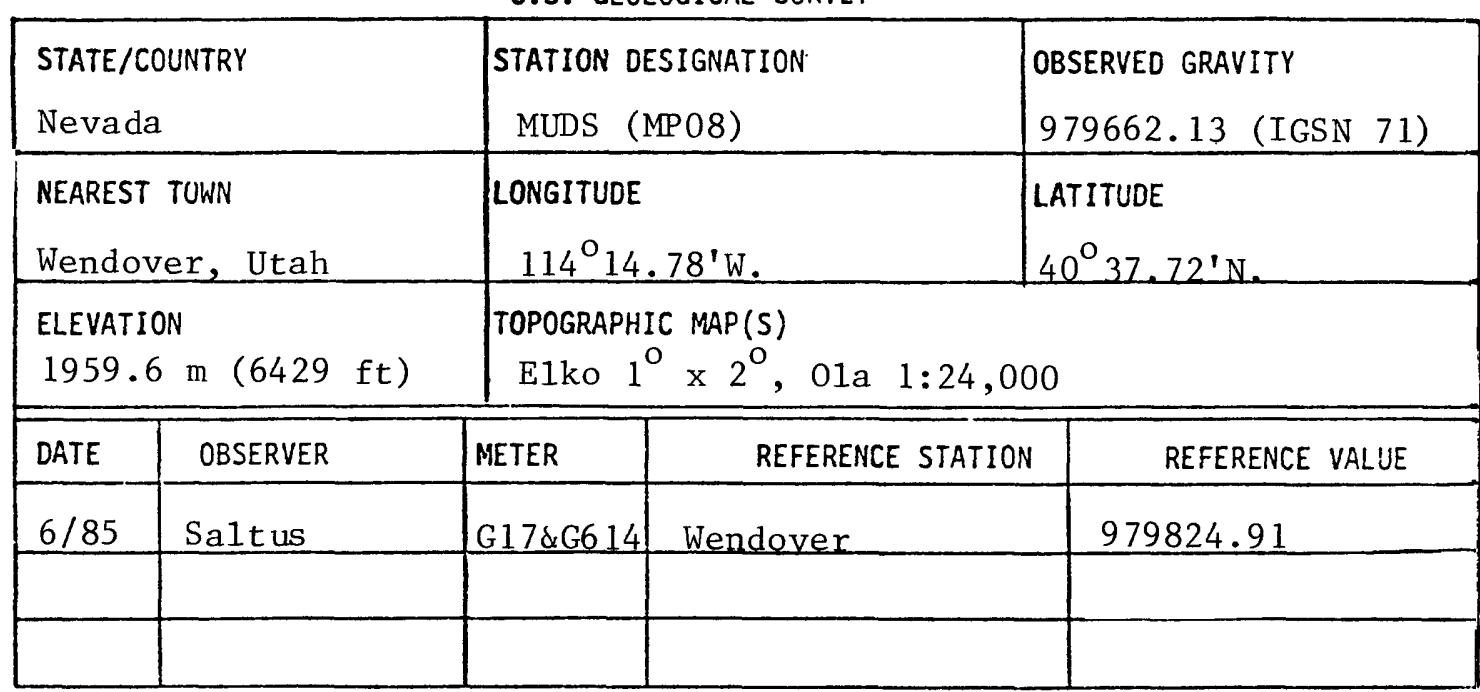

DESCRIPTION/SKETCH

The station is located at the southeast corner of the second triangle intersection to the east of Morgan Pass. This intersection is about $3 / 4 \mathrm{mile}$ south of Mud Spring. The station is $1 \mathrm{ft}$ SW of some whitewashed boulders in the center of the triangular intersection island. The station is about 27 feet north of the center of the intersection marked 6429 .

\section{TO WENDOVER}

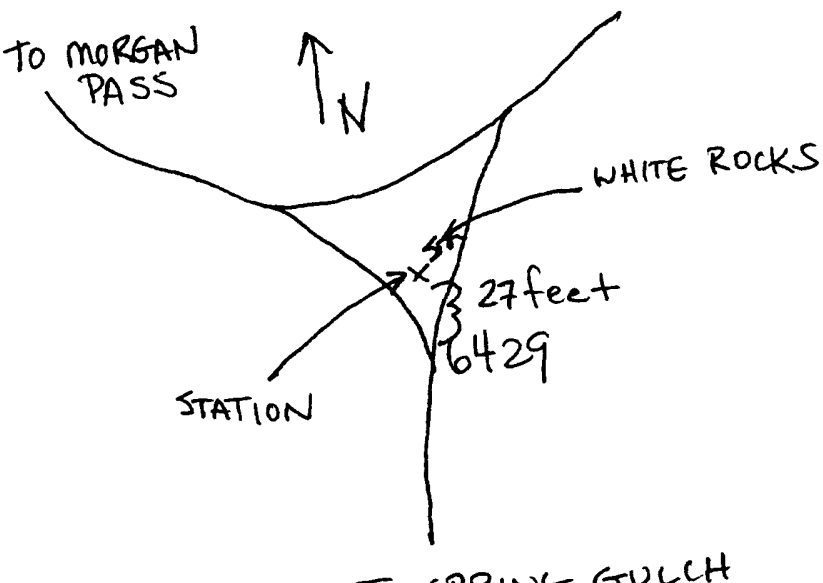

TO SPRING GULCH 
GRAVITY BASE STATION

U.S. GEOLOGICAL SURVEY

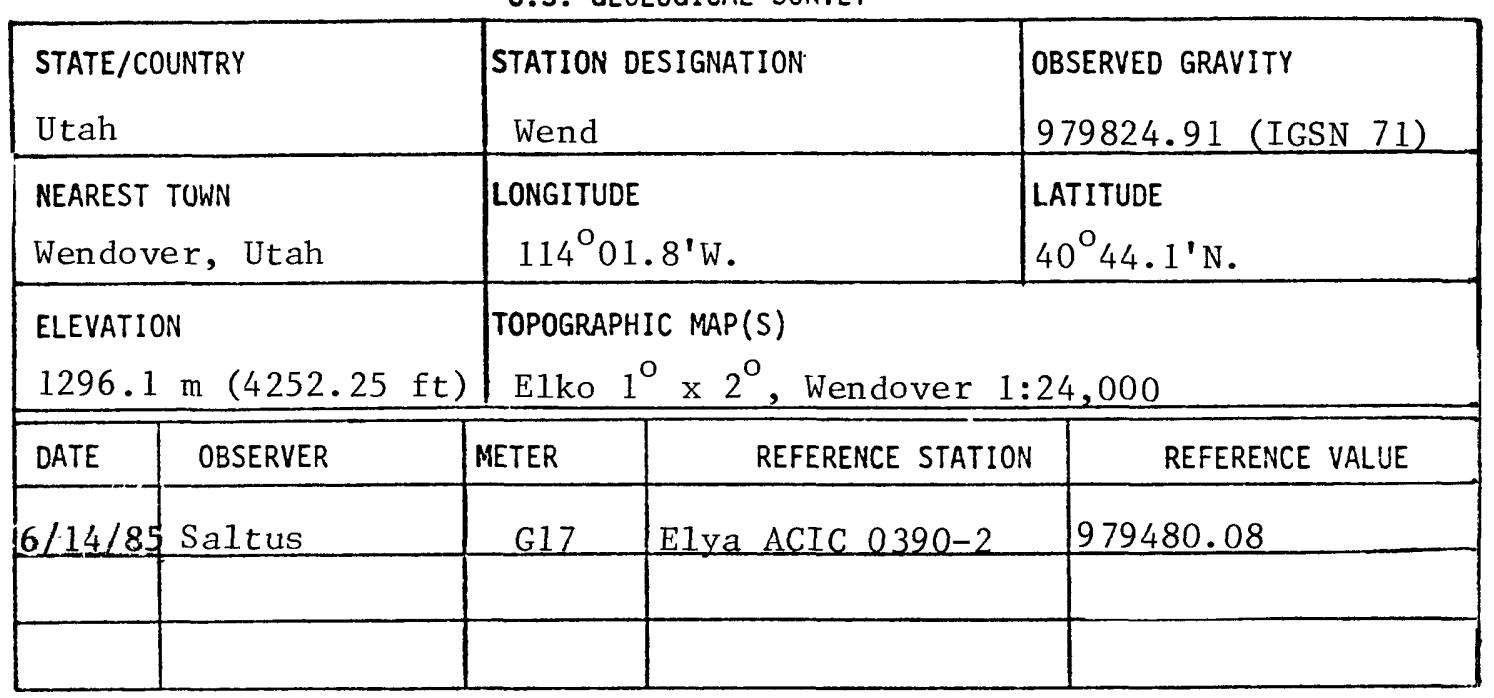

DESCRIPTION/SKETCH

This station was established on $6 / 7 / 85$ when it was discovered that original ACIC station 4646-1 had been destroyed.

The station is located near USC and GS benchmark "J334 1945", which is set in a low concrete post near the entrance to old Wendover Air Force Base (abandoned) in the southern part of Wendover, Utah. The benchmark itself is located just inside the perimeter fence of the air base. The station is located on the ground directly outside the fence from the benchmark (about $2 \mathrm{ft}$ from the benchmark). In June, 1985, the exact location of the station was marked by a piece of flat steel held in place by old railroad spikes.

WENDOVER

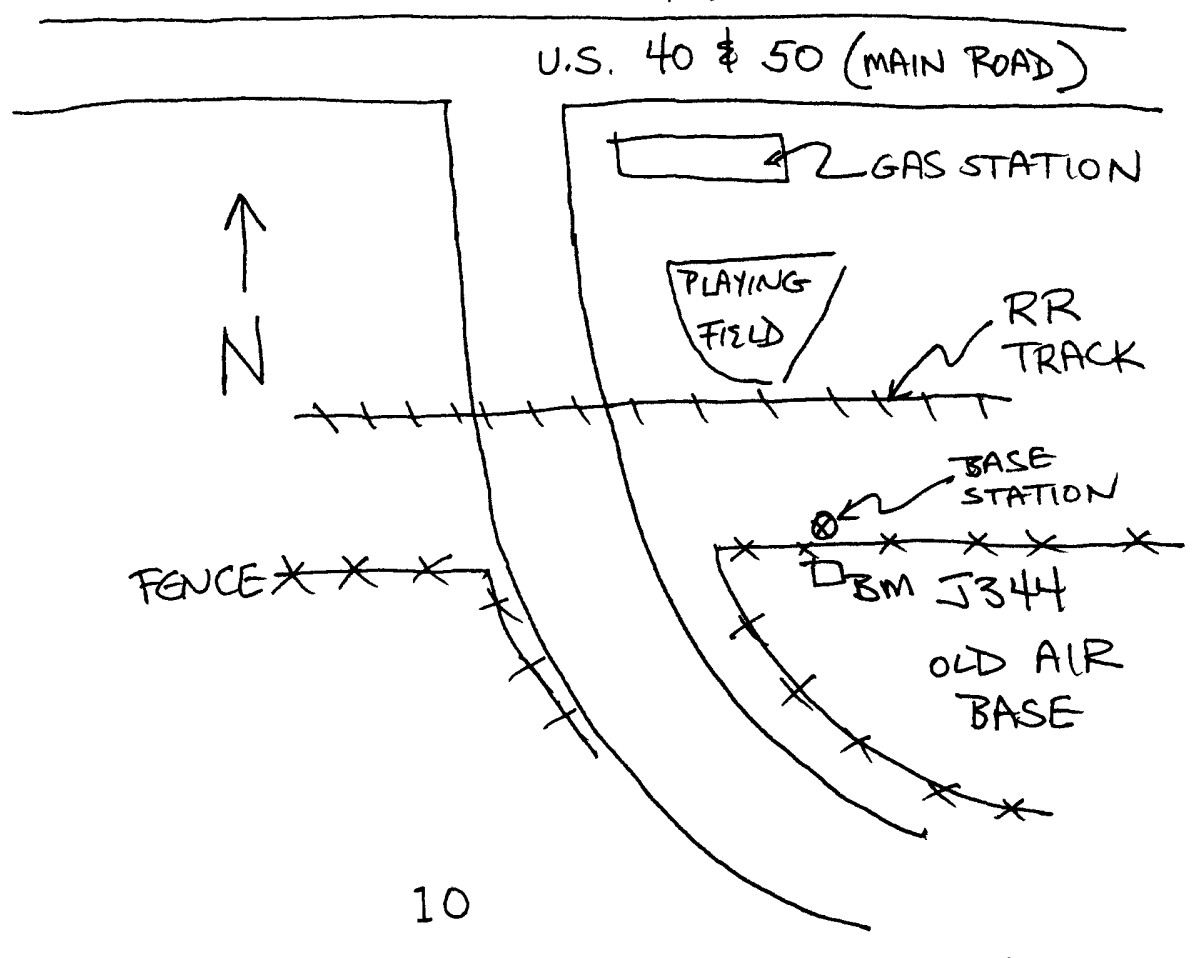




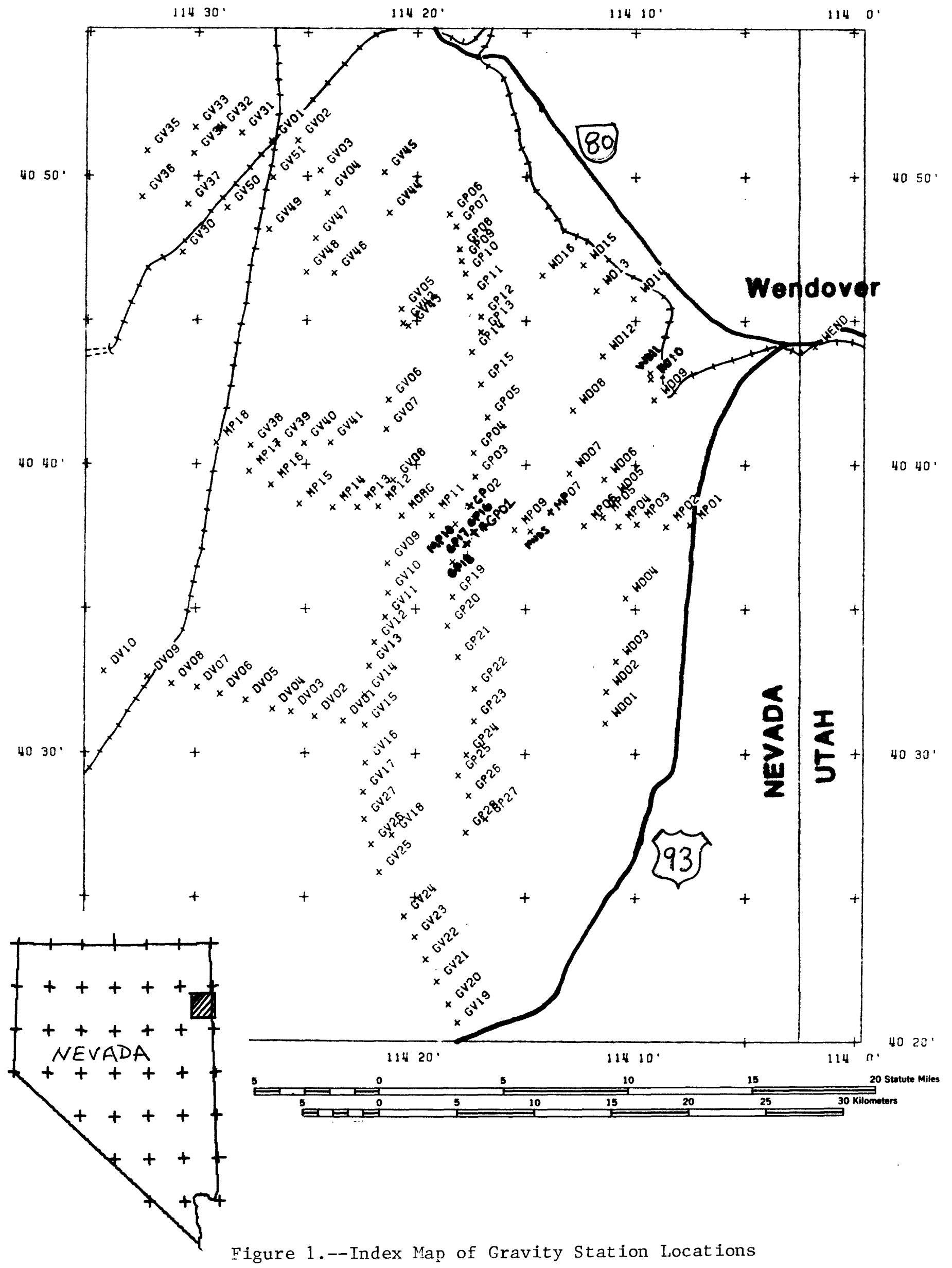


Table 1.--Data Format

Explanation of Columns in the Principal Facts Table

Sta

Station Identification

GP = Goshute Peak north-south profile

MP = Morgan Pass east-west profile

$\mathrm{DV}=$ Dolly Varden line in Steptoe Valley

$\mathrm{GV}=$ Goshute Valley stations

$W D=$ Stations on the Wendover side of the range

Latitude and Longitude

Elevation

In degrees and decimal minutes, digitized by computer from 1:24,000 scale maps.

In feet, accuracy code indicates source.

ACC

One letter and three digits indicating elevation, location, and observed gravity accuracy (see table 2 for explanation).

The remaining columns contain gravity values in milligals (mGal) to the nearest hundredth.

Observed gravity

Relative to the IGSN-71 datum.

FA

Free air anomaly.

Near

Inner zone terrain correction calculated to a distance of $590 \mathrm{~m}$ using cylindrical ring templates (when followed by an " $\mathrm{H}$ ") or estimated using 15 second digital data.

TC

Near plus digital terrain correction out to 166.7 $\mathrm{km}$ calculated using a 15 second digital terrain model.

CBA

Complete Bouguer anomaly for crustal density of $2.67 \mathrm{~g} / \mathrm{cm}^{3}$.

Isostatic residual anomaly. Based on an Airy compensation model with a $25 \mathrm{~km}$ depth to Moho at sea level, a density contrast of $0.4 \mathrm{~g} / \mathrm{cm}^{3}$ at depth, and a topographic load density of 2.67 $\mathrm{g} / \mathrm{cm}^{3}$. 
Table 2.--Accuracy Codes used in this Report

GENERAL LOCATION CODE (1st digit)

(vertical and horizontal)

Code

Station Location Method (vertical and horizontal)

A) Survey Marks (vertical) - Topographic Maps (horizontal)

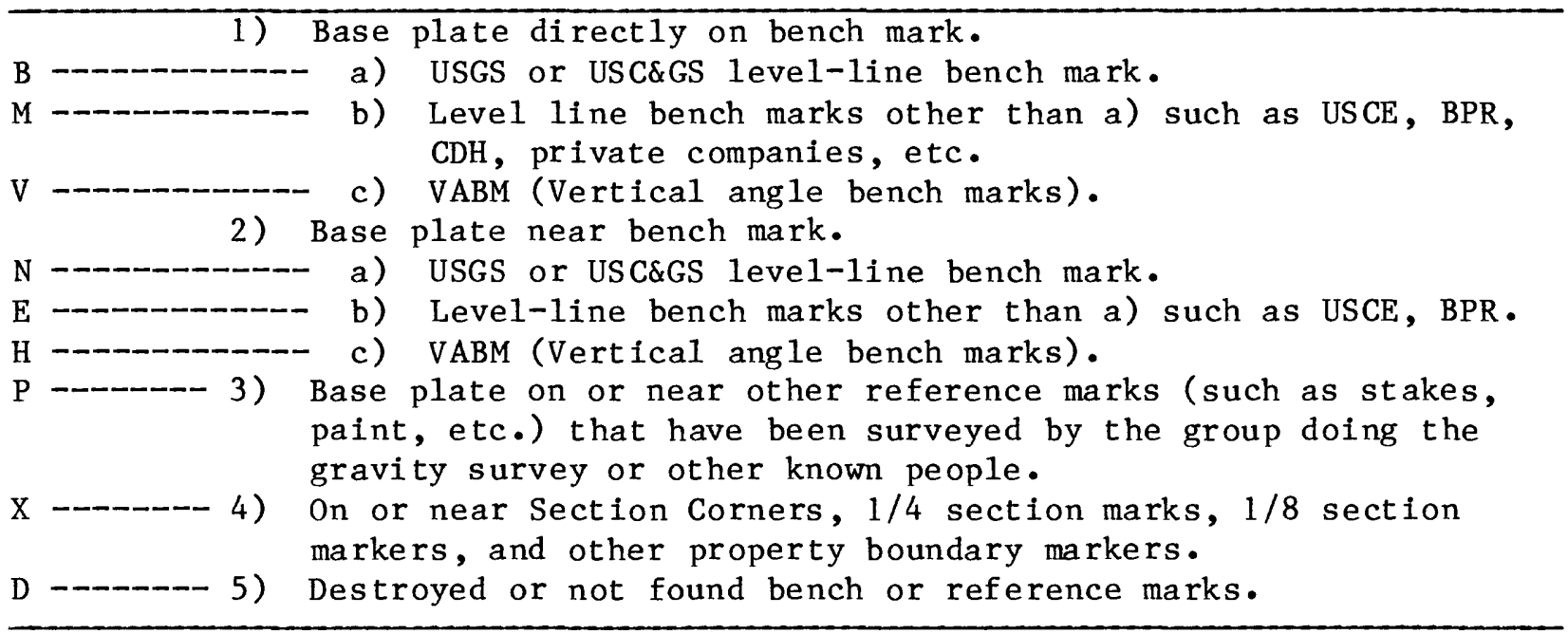

B) Topographic Map Locations (vertical and horizontal)

\begin{tabular}{ll}
\hline F - 2) & $\begin{array}{l}\text { Black spot elevations - field checked. } \\
\text { Brown spot elevations and elevations taken off original } \\
\text { manuscripts - not field checked. }\end{array}$ \\
W - 3) & Blue lake elevations \\
R - & Lake or reservoir elevations determined from leveling to bench \\
marks, and water level is determined from gauging stations. \\
C - 5) Sea level elevations. \\
Q -
\end{tabular}

C) Air Photographs (vertical and horizontal)

\begin{tabular}{ll}
\hline $\mathrm{T}-1$ Elevations determined by U.S. Geological Survey Topographic \\
Division by Kelsh plotter or least squares computer system.
\end{tabular}

D) Altimetry (vertical) - Topographic Maps (horizontal)

A- 1) lood contro1 (Leap frog, double loop, two or more altimeters,
etc.)
Poor control.

E) Special Sources

\begin{tabular}{l} 
Z 1) $\begin{array}{l}\text { Elevations determined by methods such as mobile elevation } \\
\text { recorders - horizontal control from Topo Maps. } \\
\text { Other special sources. }\end{array}$ \\
\hline
\end{tabular}

F) Unknown Elevation Sources

U - Elevation data sources unknown (this would include reference marks with unknown ties). 
Table 2.--(cont.) ELEVATION ACCURACY CODE (2nd digit)

(relative to 1929 USC \& GS mean sea leve1 datum)

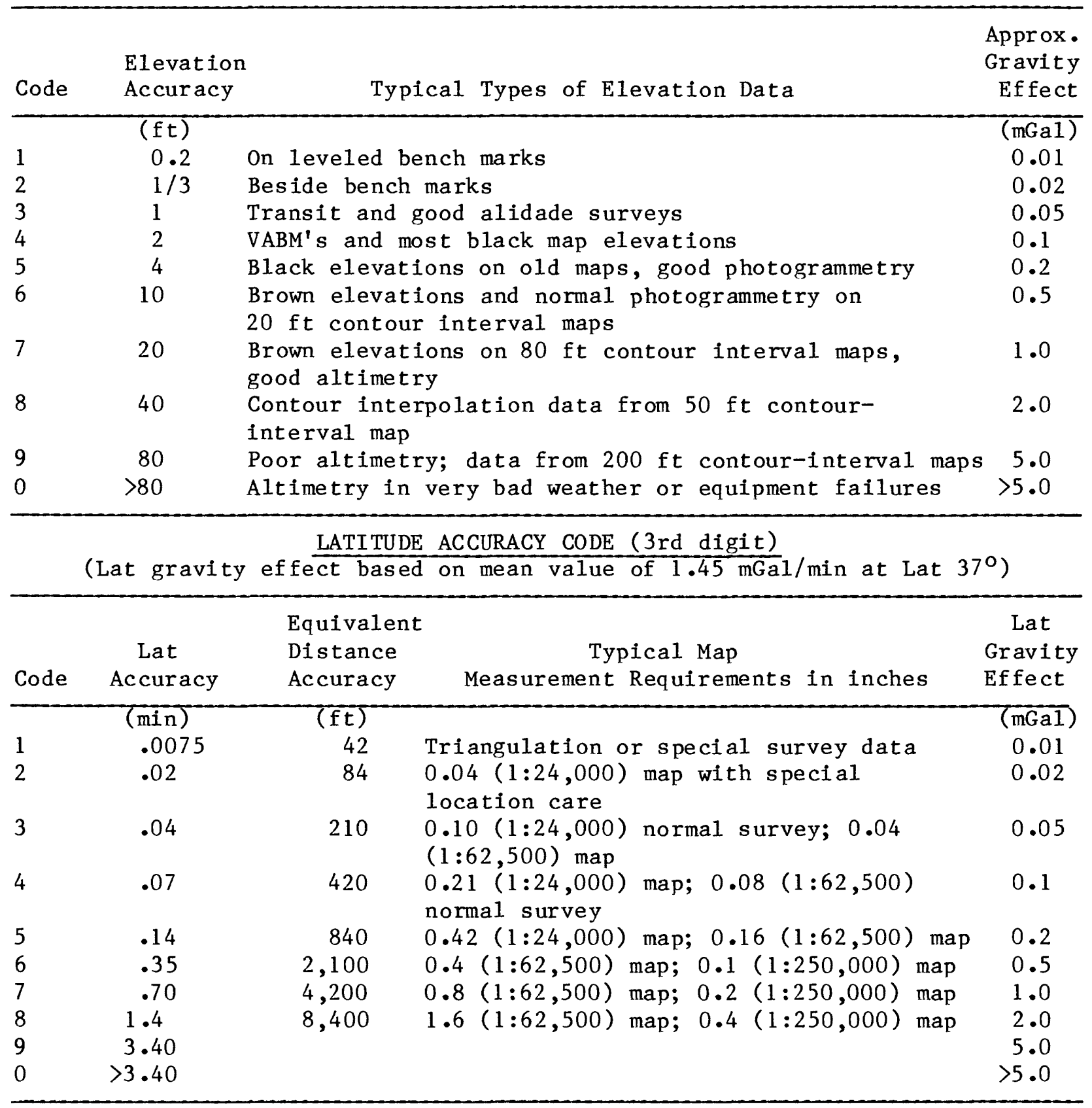

OBSERVED GRAVITY ACCURACY (4th digit)

(relative to local base)

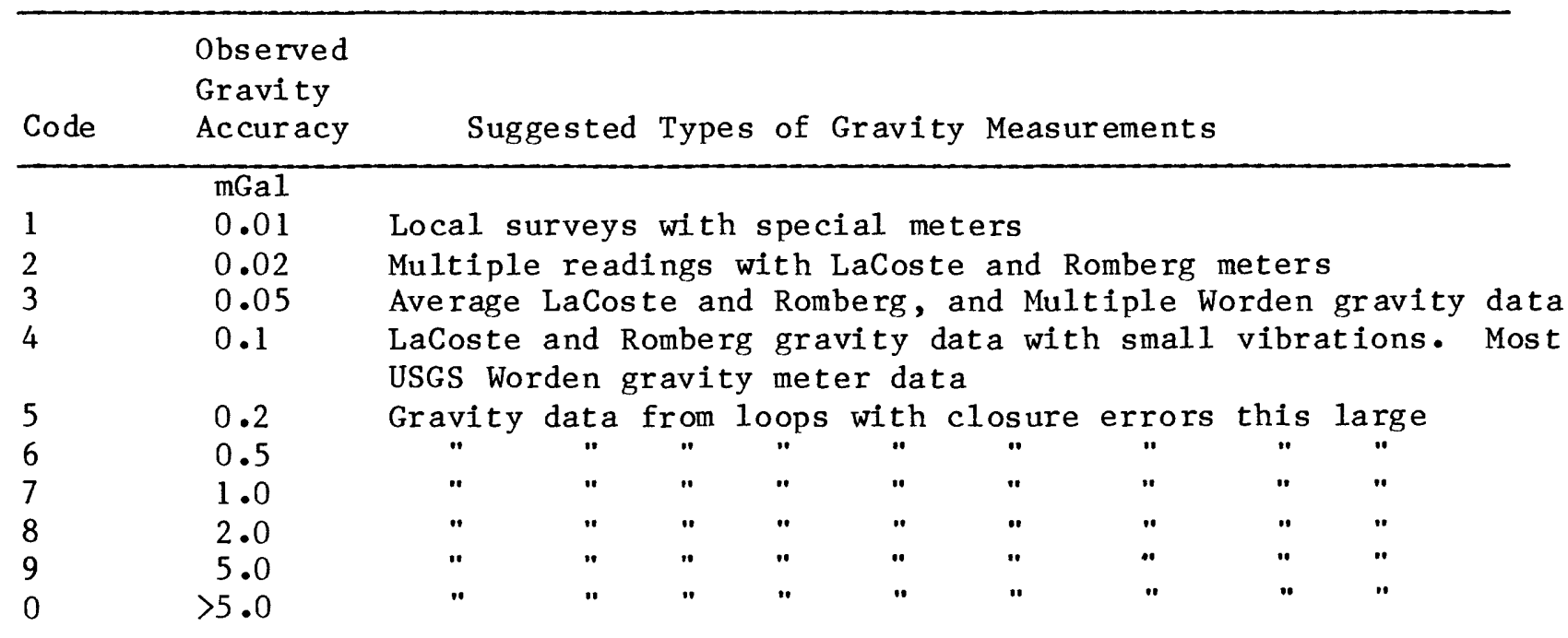


Table 3.--Principal facts for 125 gravity stations in the Goshute-Toano Range, Nevada

Base datum IGSN-71

GRS 1967 formula for theoretical gravity

Bouguer reduction density $2.67 \mathrm{~g} / \mathrm{cc}$

Sta Latitude Longitude Elev. Observed ACC FA Near TC CBA Iso deg min deg min feet gravity

GP0 $140 \quad 37.85 \quad 114 \quad 16.91 \quad 7310.0 \quad 979610.11 \quad$ A732 GP02 $40 \quad 38.44 \quad 114 \quad 17.61 \quad 7574.0 \quad 979590.03 \quad$ F433 $\begin{array}{lllllllllll}\text { GPO } 3 & 40 & 39.59 & 114 & 17.30 & 8698.0 & 979505.15 & \text { V4 } 33\end{array}$ GP04 $40 \quad 40.41 \quad 114 \quad 17.38 \quad 8107.0 \quad 979555.01 \quad$ F433 GP05 $40 \quad 41.65 \quad 114 \quad 16.75 \quad 7875.0 \quad 979575.10 \quad$ F433 GP06 $40 \quad 48.71 \quad 114 \quad 18.50 \quad 7003.0 \quad 979648.34 \quad$ F433 $\begin{array}{lllllllll}\text { GP07 } & 40 & 48.28 & 114 & 18.20 & 7196.0 & 979636.65 & \text { F433 }\end{array}$ GP08 $40 \quad 47.49 \quad 114 \quad 18.03 \quad 7618.0 \quad 979606.44 \quad$ F433 $\begin{array}{llllllllll}\text { GP09 } & 40 & 47.08 & 114 & 17.95 & 7744.0 & 979595.89 & \text { F433 }\end{array}$ GP10 $40 \quad 46.64 \quad 114 \quad 17.77 \quad 7717.0 \quad 979595.20 \quad$ F433 GP1 $140 \quad 45.85 \quad 114 \quad 17.55 \quad 7674.0 \quad 979598.43 \quad$ F433 GP12 $40 \quad 45.14 \quad 114 \quad 17.06 \quad 7822.0 \quad 979585.87 \quad$ F433 $\begin{array}{llllllllll}\text { GP1 } 340 & 44.60 & 114 & 17.05 & 7801.0 & 979588.64 & \text { F433 }\end{array}$ GP14 $40 \quad 43.91 \quad 114 \quad 17.47 \quad 7790.0 \quad 979588.68 \quad$ F433 $\begin{array}{llllllllll}\text { GP15 } & 40 & 42.78 & 114 & 17.05 & 7672.0 & 979593.12 & \text { F4 33 }\end{array}$ $\begin{array}{llllllllll}\text { GP16 } & 40 & 37.67 & 114 & 17.37 & 8018.0 & 979555.08 & \text { F433 }\end{array}$ $\begin{array}{llllllllll}\text { GP17 } & 40 & 37.27 & 114 & 17.71 & 8158.0 & 979544.92 & \text { F4 } 33\end{array}$ $\begin{array}{llllllllll}\text { GP } 18 & 40 & 36.63 & 114 & 18.33 & 8316.0 & 979529.65 & \text { F433 }\end{array}$ $\begin{array}{llllllllll}\text { GP1 } & 40 & 35.42 & 114 & 18.32 & 8693.0 & 979500.53 & \text { F433 }\end{array}$ $\begin{array}{llllllllll}\text { GP20 } & 40 & 34.42 & 114 & 18.55 & 8842.0 & 979489.54 & \text { F433 }\end{array}$ GP2 $1 \quad 40 \quad 33.34 \quad 114 \quad 18.06 \quad 7990.0 \quad 979555.92 \quad$ F433 $\begin{array}{lllllllllll}\text { GP22 } & 40 & 32.24 & 114 & 17.31 & 8144.0 & 979543.70 & \text { F433 }\end{array}$ $\begin{array}{lllllllll}\text { GP2 } 3 & 40 & 31.13 & 114 & 17.33 & 8976.0 & 979479.42 & \text { F433 }\end{array}$ GP24 $40 \quad 29.96 \quad 114 \quad 17.68 \quad 9609.0 \quad 979431.18 \quad$ V433 120 GP2 $5 \quad 40 \quad 29.24 \quad 114 \quad 18.03 \quad 9016.0 \quad 979476.39 \quad$ F433 111 $\begin{array}{llllllllll}\text { GP26 } & 40 & 28.54 & 114 & 17.56 & 8241.0 & 979532.45 & \text { F433 }\end{array}$ $\begin{array}{llllllllll}\text { GP27 } & 40 & 27.73 & 114 & 16.78 & 8281.0 & 979528.00 & \text { F433 }\end{array}$ $\begin{array}{lllllllll}\text { GP28 } & 40 & 27.27 & 114 & 17.69 & 7956.0 & 979546.22 & \text { F433 }\end{array}$ $\begin{array}{lllllllll}\text { MP0 } 1 & 40 & 37.93 & 114 & 7.51 & 4640.0 & 979765.89 & \text { N233 } & -23\end{array}$ $\begin{array}{llllllllll}\text { MP02 } & 40 & 37.87 & 114 & 8.60 & 4735.0 & 979762.22 & \text { A733 } & -1\end{array}$ $\begin{array}{lllllllll}\text { MP0 } 3 & 40 & 37.96 & 114 & 9.93 & 4932.0 & 979752.24 & \text { A733 }\end{array}$ $\begin{array}{lllllllll}\text { MP0 } 4 & 40 & 37.89 & 114 & 10.78 & 5172.0 & 979741.89 & \text { F433 }\end{array}$ $\begin{array}{lllllllll}\text { MP0 } 5 & 40 & 38.20 & 114 & 11.48 & 5352.0 & 979728.06 & \text { F4 33 }\end{array}$ $\begin{array}{llllllllll}\text { MP06 } & 40 & 37.91 & 114 & 12.33 & 5615.0 & 979713.32 & \text { F433 }\end{array}$ $\begin{array}{lllllllllll}\text { MP07 } & 40 & 38.33 & 114 & 13.93 & 6038.0 & 979686.05 & \text { F433 }\end{array}$ $\begin{array}{lllllllll}\text { MP09 } & 40 & 37.75 & 114 & 15.51 & 6608.0 & 979653.86 & \text { F433 }\end{array}$ $\begin{array}{lllllllll}\text { MP10 } & 40 & 37.96 & 114 & 18.20 & 6548.0 & 979656.10 & \text { A733 }\end{array}$ $\begin{array}{llllllllll}\text { MP11 } & 40 & 38.26 & 114 & 19.28 & 6086.0 & 979684.12 & \text { A733 }\end{array}$ $\begin{array}{llllllllll}\text { MP1 } & 40 & 38.56 & 114 & 21.72 & 5617.0 & 979704.65 & \text { N233 }\end{array}$ MP13 $40 \quad 38.54 \quad 114 \quad 22.66 \quad 5595.0 \quad 979695.42 \quad$ A733 $\begin{array}{llll}72.05 & 0.58 \mathrm{H} \quad 5.51 & -173.28\end{array}$ $\begin{array}{lllll}75.89 & 3.34 \mathrm{H} & 10.62 & -173.32\end{array}$ $94.896 .16 \mathrm{H} 28.78-174.39$ $88.01 \quad 3.21 \mathrm{H} \quad 15.61-174.34$ $84.453 .47 \mathrm{H} \quad 13.60-172.02$ $\begin{array}{llll}65.24 & 2.32 \mathrm{H} & 6.74 & -168.39\end{array}$ $\begin{array}{lllll}72.32 & 1.62 \mathrm{H} & 6.74 & -167.88\end{array}$ $\begin{array}{llll}82.95 & 2.33 \mathrm{H} & 10.82 & -167.56\end{array}$ $84.842 .64 \mathrm{H} \quad 12.78-167.99$ $82.27 \quad 3.41 \mathrm{H} \quad 13.31 \quad-169.11$ $82.64 \quad 4.46 \mathrm{H} \quad 13.46-167.13$ $\begin{array}{llllll}85.04 & 3.81 \mathrm{H} & 14.41 & -168.82\end{array}$ $86.643 .15 \mathrm{H} \quad 12.12-168.79$ $\begin{array}{lllll}86.68 & 1.83 \mathrm{H} & 9.99 & -170.51\end{array}$ $81.72 \quad 2.60 \mathrm{H} \quad 10.01-171.44$ $\begin{array}{lllll}83.80 & 4.61 & 17.74 & -173.40\end{array}$ $87.40 \quad 4.37 \mathrm{H} \quad 19.40-172.91$ $87.92 \quad 5.68 \mathrm{H} \quad 23.44-173.71$ $96.026 .74 \mathrm{H} 28.73-173.15$ $100.51 \quad 3.18 \mathrm{H} 27.01-175.43$ $\begin{array}{lllll}88.46 & 1.23 \mathrm{H} & 10.58 & -174.94\end{array}$ $\begin{array}{lllll}92.35 & 2.86 \mathrm{H} & 13.30 & -173.57\end{array}$

12.13 12.39 10.95 10.97 12.82 17.06 17.41 17.63 17.16 15.96 17.86 15.93 15.99 14.54 13.48 12.26 12.99 12.63 13.35 11.37 11.83 12.99 12.11 13.99 14.92 15.72 14.86 14.09 $-2.71$ $84.61 \quad 4.18 \mathrm{H} \quad 14.37-173.85$ $\begin{array}{llll}83.21 & 0.01 \mathrm{H} & 0.55 & -182.28\end{array}$ $\begin{array}{llll}-17.86 & 1.16 \mathrm{H} & 1.89 & -178.85\end{array}$ $\begin{array}{llll}-9.46 & 0.05 \mathrm{H} & 1.06 & -178.02\end{array}$ $\begin{array}{lllll}2.85 & 0.06 \mathrm{H} & 1.34 & -173.64\end{array}$ $\begin{array}{lllll}5.48 & 0.07 \mathrm{H} & 1.51 & -177.00\end{array}$ $\begin{array}{lllll}15.89 & 0.12 \mathrm{H} & 1.90 & -175.19\end{array}$ $27.740 .39 \mathrm{H} \quad 2.97-176.73$ $49.98 \quad 0.12 \mathrm{H} \quad 2.89-174.02$ $46.271 .11 \mathrm{H}$ $30.430 .31 \mathrm{H}$ $6.440 .01 \mathrm{H}$ $-4.830 .01 \mathrm{H}$ MP14 $40 \quad 38.52 \quad 114 \quad 23.79 \quad 5590.0 \quad 979687.72 \quad$ F433 $-12.97 \quad 0.01 \mathrm{H}$ $\begin{array}{llllllllllllll}\text { MP15 } & 40 & 38.65 & 114 & 25.30 & 5587.0 & 979679.10 & \text { A733 } & -22.07 & 0.01 \mathrm{H}\end{array}$ $\begin{array}{lllllllllll}\text { MP16 } & 40 & 39.30 & 114 & 26.61 & 5586.0 & 979677.11 & \text { A733 } & -25.12 & 0.01 \mathrm{H}\end{array}$ MP17 $40 \quad 39.77 \quad 114 \quad 27.60 \quad 5585.0 \quad 979677.09 \quad$ A733 $\quad-25.93 \quad 0.01 \mathrm{H}$ $\begin{array}{llllllllllll}\text { MP18 } & 40 & 40.75 & 114 & 29.10 & 5583.0 & 979685.91 & \mathrm{~N} 233 & -18.76 & 0.01 \mathrm{H}\end{array}$ $\begin{array}{llllllllllll}\text { DV0 } & 40 & 31.14 & 114 & 23.27 & 5593.0 & 979672.26 & \text { N233 } & -17.16 & 0.00\end{array}$ $\begin{array}{llllllllllll}\text { DV02 } & 40 & 31.29 & 114 & 24.56 & 5595.0 & 979666.43 & \mathrm{~N} 233 & -23.02 & 0.01\end{array}$ $\begin{array}{llllllllllll}\text { DV03 } & 40 & 31.45 & 114 & 25.63 & 5596.0 & 979665.23 & \mathrm{~N} 233 & -24.37 & 0.02\end{array}$

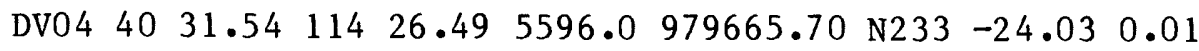
$\begin{array}{lllllllllll}\text { DV05 } & 40 & 31.84 & 114 & 27.70 & 5596.0 & 979668.02 & \text { N233 } & -22.15 & 0.01\end{array}$
$4.99-173.59$

$3.20-175.45 \quad 11.32$

$1.00-185.61 \quad 2.46$

$0.68-196.45-7.88$

$0.49-204.61-15.46$

$0.37-213.72-23.87$

$0.33-216.78-26.40$

$0.33-217.56-26.80$

$0.40-210.25-18.99$

$0.99-208.40-18.24$

$0.59-214.73-23.97$

$0.43-216.27-25.04$

$0.35-216.01-24.40$

$0.31-214.18-22.07$ 
Table 3.--Principal facts for 125 gravity stations in the Goshute-Toano Range, Nevada (cont.)

Base datum IGSN-71

GRS 1967 formula for theoretical gravity

Bouguer reduction density $2.67 \mathrm{~g} / \mathrm{cc}$

Sta Latitude Longitude Elev Observed ACC FA Near TC CBA Iso deg min deg min feet gravity

$\begin{array}{lllllllllllll}\text { DV0 } 6 & 40 & 32.05 & 114 & 28.88 & 5596.0 & 979671.51 & \mathrm{~N} 233 & -18.98 & 0.02\end{array}$ $\begin{array}{lllllllllllll}\text { DV07 } & 40 & 32.27 & 114 & 29.92 & 5600.0 & 979673.83 & \text { N233 } & -16.61 & 0.02\end{array}$ $\begin{array}{lllllllllll}\text { DV0 } 8 & 40 & 32.39 & 114 & 31.08 & 5599.0 & 979677.36 & \text { N233 } & -13.35 & 0.03\end{array}$ $\begin{array}{llllllllll}\text { DV09 } & 40 & 32.62 & 114 & 32.18 & 5597.0 & 979682.68 & \text { N233 }\end{array}$ DV10 $40 \quad 32.82 \quad 114 \quad 34.16 \quad 5768.0 \quad 979684.00 \quad$ N233 GV01 $40 \quad 51.25 \quad 114 \quad 26.65 \quad 5591.0 \quad 979718.12 \quad$ F433 GV02 $40 \quad 51.26 \quad 114 \quad 25.44 \quad 5595.0 \quad 979719.15 \quad$ F433 $\begin{array}{lllllllllll}\text { GV03 } & 40 & 50.21 & 114 & 24.42 & 5598.0 & 979717.45 & \text { F433 }\end{array}$ GV0 $4 \quad 40 \quad 49.44 \quad 114 \quad 24.08 \quad 5597.0 \quad 979715.96 \quad$ F433 GV05 $40 \quad 45.41 \quad 114 \quad 20.69 \quad 5772.0 \quad 979718.13 \quad$ F433 GV06 $40 \quad 42.26 \quad 114 \quad 21.25 \quad 5696.0 \quad 979712.83 \quad$ F433 $\begin{array}{llllllllll}\text { GV07 } & 40 & 41.24 & 114 & 21.35 & 5715.0 & 979708.74 & \text { F433 }\end{array}$ $\begin{array}{lllllllllll}\text { GV08 } & 40 & 39.48 & 114 & 21.05 & 5698.0 & 979708.78 & \text { F433 }\end{array}$ $\begin{array}{llllllllll}\text { GV09 } & 40 & 36.59 & 114 & 21.29 & 5609.0 & 979701.16 & \text { N233 }\end{array}$ $\begin{array}{llllllllll}\text { GV10 } & 40 & 35.57 & 114 & 21.25 & 5615.0 & 979700.76 & \text { N233 }\end{array}$ GV11 $40 \quad 34.73 \quad 114 \quad 21.39 \quad 5614.0 \quad 979700.06 \quad$ N233 GV12 $40 \quad 33.85 \quad 114 \quad 21.87 \quad 5614.0 \quad 979696.30$ N233 GV13 $40 \quad 33.03 \quad 114 \quad 22.10 \quad 5612.0 \quad 979692.36$ N233 GV13 $40 \quad 33.03 \quad 114 \quad 22.10 \quad 5612.0 \quad 979692.44 \quad \mathrm{~N} 233$ $\begin{array}{llllllllll}\text { GV14 } & 40 & 32.04 & 114 & 22.30 & 5609.0 & 979689.61 & \text { N233 }\end{array}$ GV15 $40 \quad 31.00 \quad 114 \quad 22.29 \quad 5606.0 \quad 979686.47 \quad$ N232 $\begin{array}{lllllllll}\text { GV16 } & 40 & 29.70 & 114 & 22.26 & 5665.0 & 979686.88 & \text { N233 }\end{array}$ $\begin{array}{lllllllllll}\text { GV17 } & 40 & 28.67 & 114 & 22.35 & 5640.0 & 979687.89 & \text { N233 }\end{array}$ GV $18 \quad 40 \quad 27.18 \quad 114 \quad 21.04 \quad 5741.0 \quad 979681.21 \quad$ C733 $\begin{array}{llllllllll}\text { GV19 } & 40 & 20.66 & 114 & 18.02 & 5630.0 & 979675.91 & \text { N233 }\end{array}$ $\begin{array}{lllllllll}\text { GV20 } & 40 & 21.29 & 114 & 18.43 & 5610.0 & 979674.06 & \text { N233 }\end{array}$ GV21 $40 \quad 22.09 \quad 114 \quad 18.97 \quad 5605.0 \quad 979671.98 \quad$ N233 $\begin{array}{llllllllll}\text { GV22 } & 40 & 22.86 & 114 & 19.48 & 5602.0 & 979671.20 & \text { N233 }\end{array}$ GV23 $40 \quad 23.63 \quad 114 \quad 19.99 \quad 5601.0 \quad 979673.97 \quad$ N233 GV24 $40 \quad 24.36 \quad 114 \quad 20.48 \quad 5601.0 \quad 979675.19 \quad$ N233 GV25 $40 \quad 25.88 \quad 114 \quad 21.60 \quad 5610.0 \quad 979678.63$ N233 $\begin{array}{lllllllll}\text { GV26 } & 40 & 26.85 & 114 & 21.97 & 5605.0 & 979681.92 & \text { N233 }\end{array}$ $\begin{array}{llllllllll}\text { GV27 } & 40 & 27.73 & 114 & 22.30 & 5610.0 & 979683.18 & \text { N233 }\end{array}$ $\begin{array}{lllllllllll}\text { GV30 } & 40 & 47.38 & 114 & 30.69 & 5582.0 & 979703.09 & \text { F433 }\end{array}$ GV31 $40 \quad 51.52 \quad 114 \quad 28.02 \quad 5584.0 \quad 979715.63 \quad$ F433 GV32 $40 \quad 51.66 \quad 114 \quad 29.04 \quad 5584.0 \quad 979713.23 \quad$ F433 GV33 $40 \quad 51.70 \quad 114 \quad 30.13 \quad 5582.0 \quad 979713.64 \quad$ F433 $\begin{array}{llllllllll}\text { GV34 } & 40 & 50.79 & 114 & 30.19 & 5582.0 & 979709.51 & \text { F433 }\end{array}$ $\begin{array}{lllllllll}\text { GV35 } & 40 & 50.88 & 114 & 32.33 & 5640.0 & 979724.02 & \text { F433 }\end{array}$ GV36 $40 \quad 49.28 \quad 114 \quad 32.55 \quad 5601.0 \quad 979725.18 \quad$ F433 GV37 $40 \quad 49.0411430 .45 \quad 5581.0 \quad 979704.01 \quad$ F433 $\begin{array}{llllllllllll}\text { GV38 } & 40 & 40.67 & 114 & 27.53 & 5585.0 & 979681.67 & \text { N233 }\end{array}$ GV39 $40 \quad 40.70 \quad 114 \quad 26.30 \quad 5587.0 \quad 979683.24 \quad$ N233 GV40 $40 \quad 40.74 \quad 114 \quad 25.11 \quad 5587.0 \quad 979689.14$ N233 GV41 $40 \quad 40.77 \quad 114 \quad 23.90 \quad 5590.0 \quad 979699.75$ N233 GV42 $40 \quad 44.91 \quad 114 \quad 20.60 \quad 5604.0 \quad 979714.37 \quad$ N233 GV43 $40 \quad 44.82 \quad 114 \quad 20.36 \quad 5810.0 \quad 979714.22 \quad$ F433 GV44 $40 \quad 48.76 \quad 114 \quad 21.25 \quad 5822.0 \quad 979719.23 \quad$ F433 GV45 $40 \quad 50.15 \quad 114 \quad 21.49 \quad 5836.0 \quad 979712.28 \quad$ F433 GV46 $40 \quad 46.65 \quad 114 \quad 23.76 \quad 5602.0 \quad 979714.64 \quad$ F433

$$
-8.560 .00
$$
$8.53 \quad 0.01$ $-1.460 .01$ $-0.07 \quad 0.00$ $\begin{array}{lll}0.08 & 0.00\end{array}$ $-0.360 .00$ $\begin{array}{lll}24.28 & 0.03\end{array}$ 16.530 .01 $\begin{array}{lll}15.74 & 0.02\end{array}$ 16.810 .09 $\begin{array}{lll}5.13 & 0.01\end{array}$ $\begin{array}{lll}6.82 & 0.01\end{array}$ $\begin{array}{lll}7.27 & 0.01\end{array}$ $\begin{array}{lll}4.83 & 0.01\end{array}$ $1.92 \quad 0.01$ $2.00 \quad 0.01$ 0.360 .01 $\begin{array}{ll}-1.51 & 0.01\end{array}$ $\begin{array}{lll}6.38 & 0.15\end{array}$ $\begin{array}{lll}6.57 & 0.23\end{array}$ $11.60 \quad 0.03$ $\begin{array}{lll}5.57 & 0.01\end{array}$ $0.91 \quad 0.01$ $\begin{array}{lll}-2.84 & 0.01\end{array}$ $\begin{array}{lll}-5.04 & 0.01\end{array}$ $-3.520 .01$ $\begin{array}{lll}-3.38 & 0.01\end{array}$ $\begin{array}{lll}-1.35 & 0.01\end{array}$ $0.02 \quad 0.00$ 0.440 .02 $-11.56 \quad 0.00$ $\begin{array}{lll}-5.01 & 0.00\end{array}$ $\begin{array}{lll}-7.62 & 0.00\end{array}$ $\begin{array}{lll}-7.46 & 0.00\end{array}$ $-10.230 .00$ 9.590 .03 $9.48 \quad 0.01$ $\begin{array}{lll}-13.21 & 0.00\end{array}$ $\begin{array}{lll}-22.70 & 0.00\end{array}$ $\begin{array}{lll}-20.98 & 0.00\end{array}$ $-15.140 .00$ $\begin{array}{lll}-4.30 & 0.00\end{array}$ $5.47 \quad 1.87$ $\begin{array}{lll}24.81 & 0.27\end{array}$ $\begin{array}{lll}25.07 & 0.03\end{array}$ $\begin{array}{lll}17.37 & 0.03\end{array}$ 2.960 .00
$0.30-211.01-18.45$

$0.31-208.77-15.81$

$0.37-205.42-12.00$

$0.43-200.50-6.70$

$\begin{array}{lll}0.70 & -188.98 \quad 5.52\end{array}$

$\begin{array}{lll}0.31 & -193.31 & -3.77\end{array}$

$0.33-192.04-3.05$

$0.37-191.95-3.42$

$0.38-192.34 \quad-3.96$

$\begin{array}{lll}1.30 & -172.77 \quad 14.07\end{array}$

$1.13-178.09 \quad 9.30$

$1.07-179.59 \quad 7.95$

$\begin{array}{lll}1.61 & -177.40 \quad 10.20\end{array}$

$1.47-186.18 \quad 1.96$

$1.78-184.39 \quad 3.88$

$1.80-183.88 \quad 4.62$

$\begin{array}{lll}1.41 & -186.71 & 2.19\end{array}$

$1.36-189.60-0.43$

$1.36-189.52-0.35$

$1.46-190.96-1.49$

$1.88-192.31-2.63$

$2.46-185.85 \quad 4.09$

$2.12-185.15 \quad 5.08$

$\begin{array}{lll}1.82 & -183.87 \quad 6.05\end{array}$

$0.75-187.17 \quad 3.10$

$0.73-191.17-0.90$

$0.78-194.70 \quad-4.41$

$0.85-196.73-6.41$

$0.90-195.12-4.75$

$0.94-194.94-4.53$

$\begin{array}{lll}0.95 & -193.21 & -2.66\end{array}$

$1.09-191.53-1.04$

$\begin{array}{llll}1.33 & -191.04 & -0.62\end{array}$

$0.36-203.05-11.74$

$0.33-196.61-6.51$

$\begin{array}{lll}0.40 & -199.15 & -8.63\end{array}$

$\begin{array}{lll}0.52 & -198.79 & -7.83\end{array}$

$\begin{array}{llll}0.46 & -201.62 & -10.62\end{array}$

$1.15-183.09 \quad 8.68$

$\begin{array}{lll}0.89 & -182.13 \quad 9.76\end{array}$

$0.38-204.65-13.51$

$0.31-214.34-23.72$

$0.31-212.70-22.65$

$\begin{array}{llll}0.35 & -206.81 & -17.32\end{array}$

$\begin{array}{llll}0.44 & -195.98 & -7.06\end{array}$

$3.91-183.23 \quad 3.61$

$\begin{array}{lll}1.94 & -172.90 & 13.81\end{array}$

$\begin{array}{lll}0.94 & -174.04 & 12.94\end{array}$

$0.82-182.35 \quad 4.73$

$0.39-189.19-0.85$ 
Table 3.--Principal facts for 125 gravity stations in the Goshute-Toano Range, Nevada (cont.)

Base datum IGSN-71

GRS 1967 formula for theoretical gravity

Bouguer reduction density $2.67 \mathrm{~g} / \mathrm{cc}$

Sta Latitude Longitude Elev observed ACC FA Near TC CBA Iso deg min deg min feet gravity

GV47 $40 \quad 47.86 \quad 114 \quad 24.615591 .0 \quad 979713.32 \quad$ F433 GV48 $40 \quad 46.68 \quad 114 \quad 25.03 \quad 5590.0 \quad 979724.31 \quad$ F433 GV49 $40 \quad 48.16 \quad 114 \quad 26.75 \quad 5586.0 \quad 979705.83 \quad$ F433

$-1.200 .00$ 11.450 .06 GV50 $40 \quad 48.91 \quad 114 \quad 28.66 \quad 5584.0 \quad 979704.18 \quad F 433 \quad-12$ GV51 $40 \quad 49.97 \quad 11426.56 \quad 5588.0 \quad 979713.42 \quad$ F433 $\begin{array}{llllllllll}\text { WD01 } & 40 & 31.07 & 114 & 11.35 & 5590.0 & 979702.82 & \text { F433 }\end{array}$ WD02 $40 \quad 32.15 \quad 114 \quad 11.31 \quad 5342.0 \quad 979719.33 \quad$ F433 WD03 $40 \quad 33.20 \quad 114 \quad 10.87 \quad 5175.0 \quad 979733.03 \quad$ F433 WD0 $4 \begin{array}{lllllll}40 & 35.39 & 114 & 10.44 & 5089.0 & 979741.44 & \text { F433 }\end{array}$ WD05 $40 \quad 38.84 \quad 114 \quad 11.11 \quad 5171.0 \quad 979734.36 \quad$ F433 WD06 $40 \quad 39.51 \quad 114 \quad 11.42 \quad 5177.0 \quad 979735.40 \quad$ F433 WD07 $40 \quad 39.71 \quad 114 \quad 13.01 \quad 5618.0 \quad 979712.86 \quad$ F433 WD08 $40 \quad 41.91 \quad 114 \quad 12.85 \quad 5590.0 \quad 979721.23 \quad$ F433 WD09 $40 \quad 42.25 \quad 114 \quad 9.14 \quad 4686.0 \quad 979772.22 \quad$ F433 -18 WD10 $40 \quad 42.97 \quad 114 \quad 9.31 \quad 4700.0 \quad 979775.62 \quad$ C833 WD1 $140 \quad 43.18 \quad 114 \quad 9.34 \quad 4711.0 \quad 979778.59 \quad$ F433 -1 WD12 $40 \quad 43.78 \quad 114 \quad 11.50 \quad 5195.0 \quad 979749.99 \quad$ F433 WD13 $40 \quad 46.05 \quad 114 \quad 11.79 \quad 5198.0 \quad 979758.99 \quad$ F433 WD14 $40 \quad 45.77 \quad 114 \quad 10.08 \quad 4961.0 \quad 979770.60 \quad$ F433 $\begin{array}{llllllllll}\text { WD15 } & 40 & 46.94 & 114 & 12.38 & 5164.0 & 979759.99 & \text { F433 }\end{array}$ WD16 $40 \quad 46.58 \quad 114 \quad 14.27 \quad 5581.0 \quad 979739.26 \quad$ F433 LAGE $40 \quad 3.46 \quad 114 \quad 37.05 \quad 5963.0 \quad 979606.63 \quad$ B232 MORG $40 \quad 38.25 \quad 114 \quad 20.65 \quad 5775.0 \quad 979701.16 \quad$ F432 MUDS $40 \quad 37.72 \quad 114 \quad 14.78 \quad 6429.0 \quad 979662.14 \quad$ F432 WEND $40 \quad 44.10 \quad 114 \quad 1.80 \quad 4252.0 \quad 979824.91 \quad$ N232
$-12.560 .00$

$-4.530 .00$

13.230 .04 4.820 .05 $\begin{array}{lll}1.26 & 0.03\end{array}$ $\begin{array}{lll}-1.67 & 0.11\end{array}$ $\begin{array}{ll}-6.19 & 0.03\end{array}$ $\begin{array}{lll}-5.58 & 0.04\end{array}$ $13.03 \quad 0.04$ 15.480 .21 $-18.990 .03$

$\begin{array}{lll}-15.36 & 0.09\end{array}$ $\begin{array}{lll}-11.66 & 0.10\end{array}$ $\begin{array}{lll}4.34 & 0.08\end{array}$ $10.23 \quad 0.05$ $-0.020 .06$ $\begin{array}{lll}6.71 & 0.06\end{array}$ $25.70 \quad 0.03$ $-6.840 .11$ $18.26 \quad 0.03 \mathrm{H}$ $41.490 .11 \mathrm{H}$ $-9.860 .20$
$-9.610 .00$

$\begin{array}{rrr}0.33 & -193.03 & -4.35 \\ 0.39 & -180.29 & 8.63 \\ 0.26 & -201.34 & -11.68 \\ 0.28 & -204.21 & -13.79 \\ 0.28 & -196.31 & -6.79 \\ 1.47 & -177.43 & 5.87 \\ 1.36 & -177.46 & 5.58 \\ 1.17 & -175.50 & 7.04 \\ 1.27 & -175.40 & 6.46 \\ 1.28 & -182.71 & -0.92 \\ 1.36 & -182.23 & -0.31 \\ 1.93 & -178.13 & 4.76 \\ 2.17 & -174.48 & 8.09 \\ 0.80 & -179.39 & 0.83 \\ 0.89 & -176.14 & 4.13 \\ 0.90 & -172.82 & 7.45 \\ 1.63 & -172.65 & 8.94 \\ 1.21 & -167.28 & 14.39 \\ 0.75 & -169.88 & 10.75 \\ 1.24 & -169.61 & 12.41 \\ 2.02 & -164.10 & 19.05 \\ 1.08 & -210.64 & -7.44 \\ 1.67 & -178.52 & 9.02 \\ 2.56 & -176.74 & 7.45 \\ 0.72 & -155.46 & 20.01\end{array}$

